\title{
1 Biodiversity trends are stronger in marine than terrestrial assemblages
}

2 *Shane Blowes ${ }^{1}$ and *Sarah Supp $^{2}$, Laura Antão ${ }^{3,4}$, Amanda Bates ${ }^{5}$, Helge Bruelheide ${ }^{1,6}$, Jon Chase ${ }^{1,7}$,

3 Faye Moyes ${ }^{3}$, Anne Magurran $^{3}$, Brian McGill $^{8}$, Isla Myers-Smith ${ }^{9}$, Marten Winter $^{1}$, Anne Bjorkman ${ }^{10}$,

4 Diana Bowler ${ }^{1}$, Jarrett E.K. Byrnes ${ }^{11}$, Andrew Gonzalez ${ }^{12}$, Jes Hines ${ }^{1,13}$, Forest Isbell ${ }^{14}$, Holly Jones ${ }^{15}$,

5 Laetitia M. Navarro ${ }^{1}$, Patrick Thompson ${ }^{16}$, Mark Vellend ${ }^{17}$, Conor Waldock ${ }^{18}$, Maria Dornelas ${ }^{3}$

6

$7 \quad{ }^{1}$ German Centre for Integrative Biodiversity Research (iDiv), Halle-Jena-Leipzig, Germany

$8 \quad{ }^{2}$ Data Analytics Program, Denison University, Granville, OH, USA

$9{ }^{3}$ Centre for Biological Diversity, School of Biology, University of St. Andrews, St. Andrews, UK

$10{ }^{4}$ Department of Biology and CESAM, Universidade de Aveiro, Aveiro, Portugal

$11{ }^{5}$ Department of Ocean Sciences, Memorial University of Newfoundland, Newfoundland, Canada

$12{ }^{6}$ Martin Luther University Halle-Wittenberg, Institute of Biology / Geobotany and Botanical Garden,

13 Halle (Saale), Germany

$14{ }^{7}$ Martin Luther University Halle-Wittenberg, Institute of Computer Science, Halle (Saale), Germany

$15{ }^{8}$ School of Biology and Ecology, University of Maine, Orono, ME, USA

$16{ }^{9}$ School of GeoSciences, University of Edinburgh, Edinburgh, UK

$17{ }^{10}$ Senckenberg Gesellschaft für Naturforschung, Biodiversity and Climate Research Centre (BiK-F),

18 Frankfurt am Main, Germany

$19{ }^{11}$ Department of Biology, University of Massachusetts Boston, Boston, MA, USA

$20 \quad{ }^{12}$ Department of Biology, McGill University, Montreal, Qc, Canada

$21 \quad{ }^{13}$ Leipzig University, Institute of Biology, Leipzig, Germany

$22{ }^{14}$ Department of Ecology, Evolution, and Behavior, University of Minnesota, St. Paul, MN, USA

$23{ }^{15}$ Department of Biological Sciences and Institute for the Study of the Environment, Sustainability, and 24 Energy, Northern Illinois University, DeKalb, IL, USA

$25{ }^{16}$ Department of Zoology, University of British Columbia, Vancouver, BC, Canada

$26{ }^{17}$ Département de biologie, Université de Sherbrooke, Sherbrooke, QC, Canada

$27{ }^{18}$ Ocean and Earth Science, National Oceanography Centre, University of Southampton, Southampton,

28 UK \& Life Sciences, Natural History Museum, Cromwell Road, London, UK.

$29 *$ These authors contributed equally to this work 


\section{Summary}

Human activities have fundamentally altered biodiversity. Extinction rates are elevated and model projections suggest drastic biodiversity declines. Yet, observed temporal trends in recent decades are highly variable, despite consistent change in species composition. Here, we uncover clear spatial patterns within this variation. We estimated trends in the richness and composition of assemblages in over 50,000 timeseries, to provide the most comprehensive assessment of temporal change in biodiversity across the planet to date. The strongest, most consistent pattern shows compositional change dominated by species turnover, with marine taxa experiencing up to fourfold the variation in rates of change of terrestrial taxa. Richness change ranged from no change to richness gains or losses of $\sim 10 \%$ per year, with tropical marine biomes experiencing the most extreme changes. Earth is undergoing a process of spatial reorganisation of species and, while few areas are unaffected, biodiversity change is consistently strongest in the oceans.

\section{Main Text}

Biodiversity is changing rapidly throughout the Anthropocene. Against a background of elevated extinction rates ${ }^{1,2}$, local biodiversity change results from multiple interacting drivers that influence the abundance and distribution of species. Different regions of the globe are projected to experience different trends in biodiversity change, particularly due to variation in the strength of drivers such as land use intensity ${ }^{3}$ and climate change ${ }^{4}$. There are widespread changes in the identities of species that live in any one location (species composition), whereas shifts in the numbers of species (species richness) show a mixed pattern with increasing, decreasing, or static trends ${ }^{5-8}$. However, the spatial distribution of the locations most 
54 affected is unknown. Here, we map biodiversity change, as species richness and

55 composition, to establish whether there are systematic trends in the biogeography of

56 biodiversity change. Our analysis compares marine and terrestrial realms, as well as

57 different biomes, and latitudinal bands examined as polar, temperate, and tropical

58 regions of the globe.

60 Biodiversity and its change is unevenly distributed on the planet ${ }^{9,10}$, and unevenly

61 sampled ${ }^{11-14}$. Detecting geographic variation in biodiversity trends will inform conservation prioritisation and improve estimates of global biodiversity change.

63 Moreover, quantifying this spatial distribution will help refine hypotheses about the

64 drivers of biodiversity change. The spatial distribution of drivers of biodiversity

65 change is heterogeneous ${ }^{15,16}$ and fundamentally differs between the marine and

66 terrestrial realms ${ }^{17}$. Specifically, there is more spatial overlap between climate change

67 and other drivers of change in the marine realm than in the terrestrial realm ${ }^{17}$.

68 Understanding the biogeography of biodiversity change across realms is essential for

69 reliable forecasting future change and its consequences.

71 Quantifying biogeographic patterns of biodiversity change will allow us to assess the

72 ongoing spatial re-organisation of species. This reorganisation is being driven by

73 climate change driven range shifts ${ }^{4,18}$, altered species abundance due to land-use

74 change $^{3}$ and widespread species introductions ${ }^{19}$. Local or regional richness will

75 decline when species losses exceed species gains, for example, in areas where land-

76 use intensity is high ${ }^{3}$ and/or when range sizes contract ${ }^{20}$. Conversely, local or regional

77 richness will increase when species gains exceed losses, occurring, for example, in

78 places where species are introduced ${ }^{21-23}$ and where ranges expand ${ }^{24,25}$, or when 
79 species are favoured by land-use change ${ }^{26}$. Combinations of different anthropogenic

80 drivers can lead to increases or decreases, depending on the magnitude of each

81 driver ${ }^{27}$.

83 Here, we quantified biogeographic variation in patterns of change in both species

84 richness and composition from a compilation of over 50,000 local assemblage time

85 series (ranging from 2 to 97 years; mean $=5.5$ years) across the globe. We use the

86 BioTIME database, which is currently the largest collation of assemblage time series

87 (332 studies analysed ${ }^{28}$; plus 26 other studies; see Table S1 in Dornelas et al. 2018;

88 Extended Data Fig. 1). The null expectation is that overall species richness should

89 remain largely stable even when compositional turnover is high, due to species gains

90 and losses at local scales being approximately balanced and widespread community

91 regulation ${ }^{29-31}$. Results from a number of analyses are consistent with this

92 expectation ${ }^{5-8,29}$. Yet there is scope for non-random spatial patterns within the

93 distribution of local species gains, losses and compositional change. Here, we

94 quantify variation in biodiversity change trends among realms, biomes, latitudinal

95 bands and taxa.

\section{Biodiversity trends across the globe}

98 To examine biogeographical patterns in biodiversity change we estimated trends in richness and composition change using hierarchical linear models. After standardising for spatial extent and sampling effort of each time-series, we quantified biogeographic

101 variation by examining departures from overall trends for 48 biomes and 9

102 taxonomic-habitat groupings (hereafter referred to as taxa; amphibians, benthos,

103 birds, fish, invertebrates, mammals, marine invertebrates/plants, plants, and multiple 
104 taxa) that were nested within biomes (resulting in 105 biome-taxa combinations [table

$105 \mathrm{~S} 1])$. We also examined the robustness of our biome-taxa models by fitting a second

106 set of simpler hierarchical models, where taxonomic groups were nested within

107 latitudinal bands (i.e., polar, temperate, tropical) for each realm (marine, terrestrial,

108 freshwater; [table S2] see supporting information for details).

109

110 Our results show that variation in biodiversity change is greater in the marine versus

111 the terrestrial and freshwater realms. The overall trend in richness change was not

112 statistically distinguishable from zero (Fig. 1), neither globally, nor at the biome level.

113 This result was robust to sensitivity analyses regarding time series duration and start

114 year (Extended Data Figs. 4 to 7). However, variation in departures from the overall

115 trend were almost four times greater among marine biomes (median biome departure

116 range: $-0.0014--0.0017, n=33$; Fig. 1a) compared with terrestrial and freshwater

117 biomes $(-0.0006-0.0002, n=15 ;$ Fig. $1 b)$. Thus, taxa in marine biomes frequently

118 represented extremes at both ends of the range of observed change in species richness

119 - negative trends of approximately $10 \%$ species loss per year and positive trends

120 approaching $15 \%$ species gains per year (Fig. 2a). At the taxa-level, this volatility in

121 marine species richness change meant that a higher proportion of biome-taxa

122 combinations were undergoing richness changes that differed from zero with $95 \%$

123 probability (36/78) compared with terrestrial and freshwater taxa (7/27).

124

125 Richness trends varied substantially across taxa within biomes, and this variation was

126 spatially structured, with the most pronounced trends found within tropical latitudes.

127 Negative richness trends (i.e. slope estimates with $90 \%$ credible intervals that do not

128 include zero) were present for taxa in five out of twelve marine tropical biomes, with 
129 positive trends present in three (Fig. 2a). These results were consistent with our

130 simpler hierarchical model that showed overall declines within tropical latitudes for

131 marine taxa (Fig. 2b). Locations where species losses outweigh gains could be driven

132 by range contractions, or by the loss of more specialised or thermally restricted

133 species as climate change, land use or seascape change, and other anthropogenic

134 drivers affect tropical habitats ${ }^{32,33}$. Geographic gaps from terrestrial tropical systems

135 remain in our assemblage time series data, precluding direct comparison between the

136 realms (see also Extended Data Fig. 1). The high rates of change we observed in the

137 marine tropics are consistent with predictions that tropical species will be relatively

138 sensitive to extreme heat events, because they are closer to their physiological limits,

139 resulting in biodiversity $\operatorname{loss}^{34}$. However, overexploitation, pollution, and other threats

140 are likely also contributing to biodiversity change. 


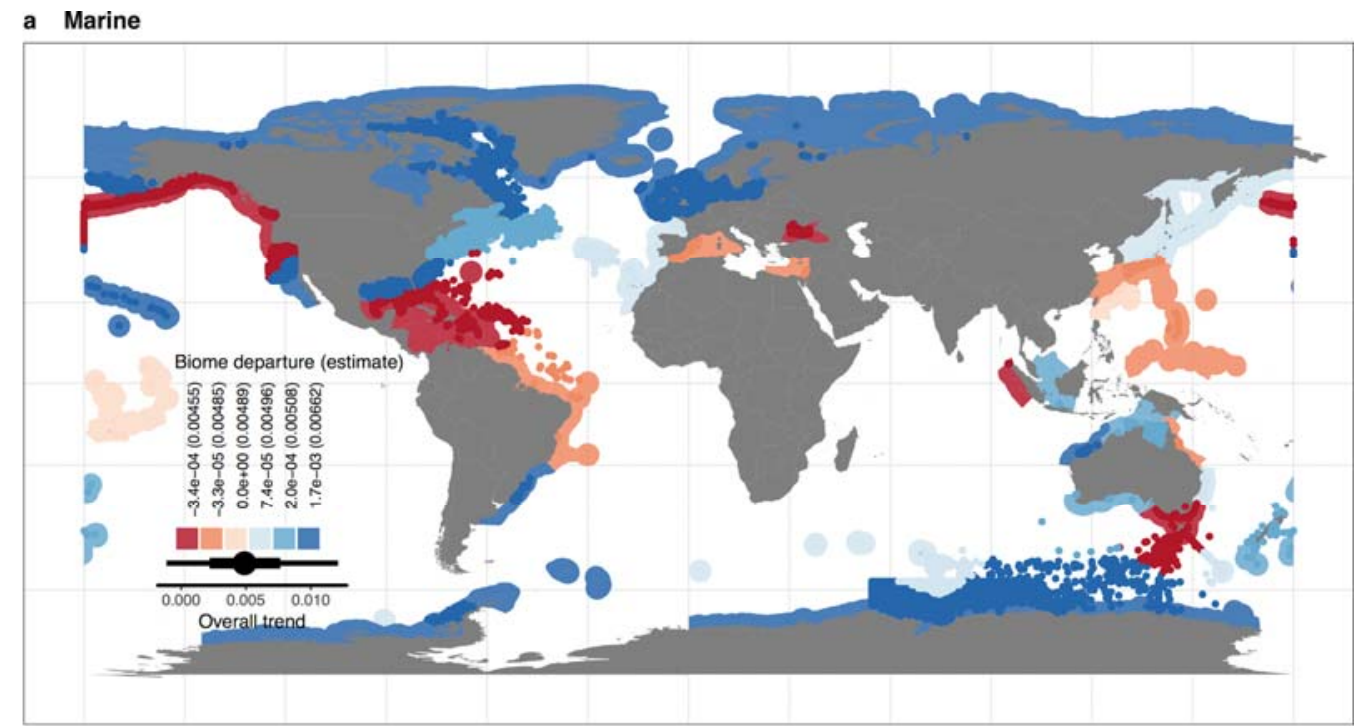

b Terrestrial/Freshwater

142

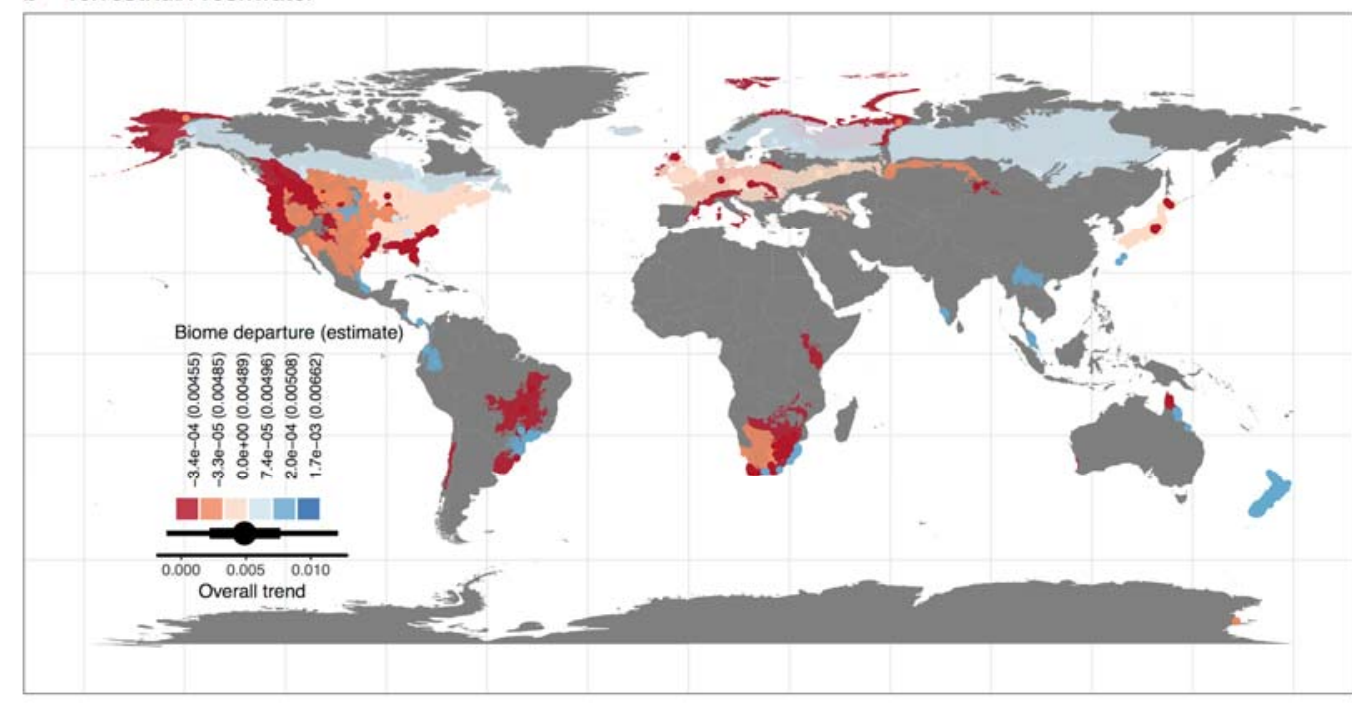

143 Figure 1. The overall trend in assemblage species richness change across biomes in the marine and

144 terrestrial realm does not differ from zero; bar depicts 50\% (thick) and 90\% (thin) credible intervals.

145 Shading represents positive (blue) and negative (red) departures from the overall trend (0.005) for each

146 biome; numbers in legend denote the departure and the biome-level (overall + departure) estimate in

147 brackets. 90\% credible intervals for all biome level estimates overlap zero. a, Marine biomes $(\mathrm{n}=33)$

148 show both positive and negative departures from the overall trend, with more negative departures in the

149 tropics, whereas there are no latitudinal trends in $\mathbf{b}$, Terrestrial $(n=10)$ and freshwater $(n=5)$ biomes.

150 Marine biomes show stronger variation in richness outcomes; particularly visible in the dark blue

151 coastal polar regions and the cluster of dark red biomes in the Caribbean and southeast coast of

152 Australia. 
153

154

a

155 Figure 2: The magnitude of species richness change is more variable among taxa in marine biomes

156 than taxa in terrestrial or freshwater biomes. The overall trend in assemblage richness change (solid

157 vertical bar) does not differ from zero (grey shading depicts the $90 \%$ credible interval) for both a, the

158 biome-taxa model, and $\mathbf{b}$, the simpler realm-climate-taxa model. Density ridges in a represent the

159 posterior distribution for the slope coefficients of each taxa-level coefficient (within each a biome); line

160 type and fill refer to the realm and taxon, respectively. Colour of biome label denotes latitudinal band

161 (light green tropical, blue green temperate and purple polar as shown in $\mathbf{b}$ ). Density ridges in $\mathbf{b}$

162 represent the posterior distributions of the slope coefficients for all taxa within a given combination of

163 realm and latitudinal band (climate) estimated with the realm-climate-taxa model.

164

165 
166 To examine changes in species composition, we partitioned total Jaccard dissimilarity,

167 calculated as the dissimilarity between the initial and each subsequent year of a time

168 series, into the additive components of turnover and nestedness ${ }^{35}$. These trends

169 describe directional compositional change relative to the initial assemblage, and the

170 decomposition examines whether changes in community composition were due to the

171 original species in assemblages being replaced by new species (turnover), or if

172 assemblages were becoming smaller subsets of themselves or growing to include new

173 species alongside the original species (nestedness). Overall, we found rates of

174 turnover were positive and much greater (0.025; 90\% credible interval: 0.021-0.029;

175 Fig. 3) than the rate of change in nestedness (0.005; 0.004-0.006; Extended Data Fig.

176 2). This means that compositional change was dominated by species replacement

177 within assemblages, with approximately $25 \%$ of species within assemblages being

178 replaced per decade. Marine biomes showed both positive and negative departures

179 from the overall trend (i.e., depending on the biome, more and less compositional

180 change over time than the global average; Fig. 3a). In contrast, the terrestrial biomes

181 showed mostly negative departures from the global average (Fig. 3b), often in highly

182 developed regions of the globe (e.g., Northeast US, Europe, Japan). Similar to our

183 finding for species richness change, variation in rates of turnover were more than 1.5

184 times greater in marine biomes (Fig. 3) and 2.5 times greater among marine taxa when

185 compared to their terrestrial and freshwater counterparts (Fig. 4). Taxa in terrestrial

186 and freshwater biomes represented 9 of the 10 lowest rates of turnover, whereas, 9 of

187 the 10 highest rates of turnover were marine taxa (Fig. 4a). Fish in marine tropical

188 biomes represented both ends of the spectrum, from among the lowest turnover rates

189 (Tropical Southwestern Atlantic) to the highest observed turnover rate (Northwest

190 Australian Shelf). 
bioRxiv preprint doi: https://doi.org/10.1101/457424; this version posted October 30, 2018. The copyright holder for this preprint (which was not certified by peer review) is the author/funder, who has granted bioRxiv a license to display the preprint in perpetuity. It is made available under aCC-BY-ND 4.0 International license.

191

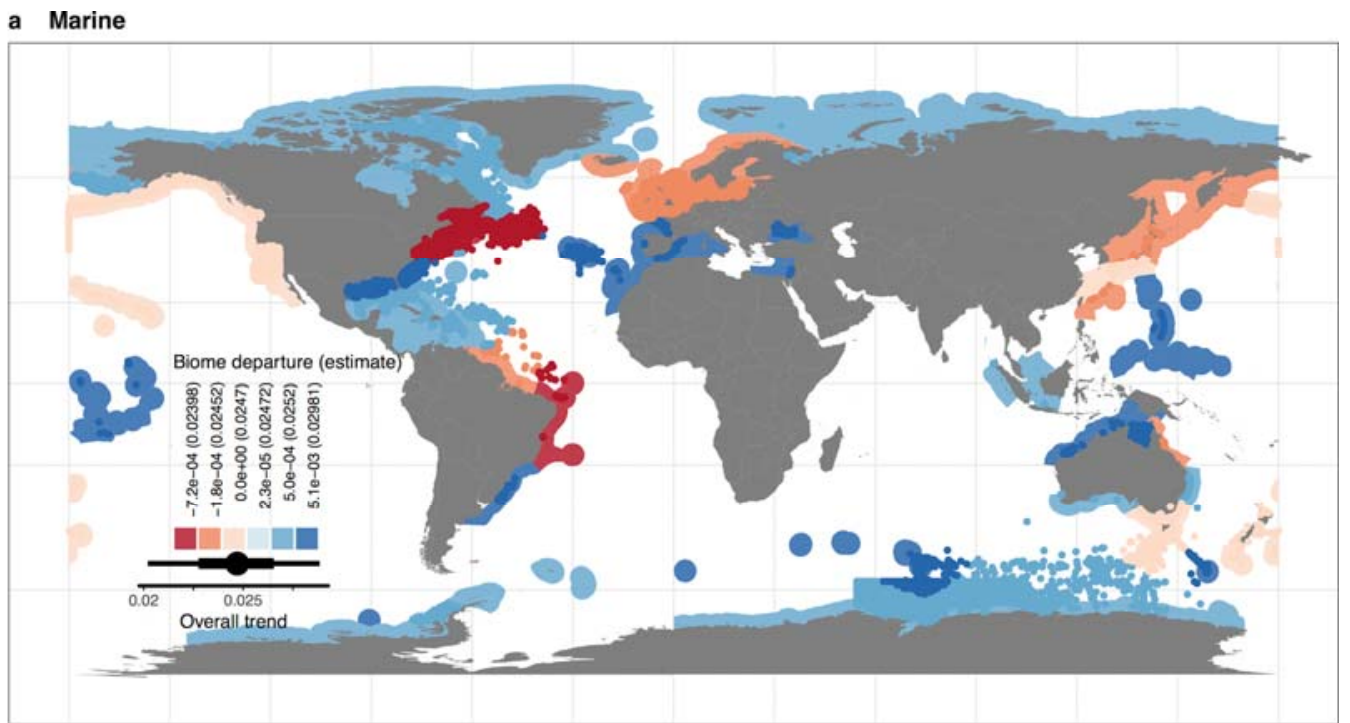

b Terrestrial/Freshwater

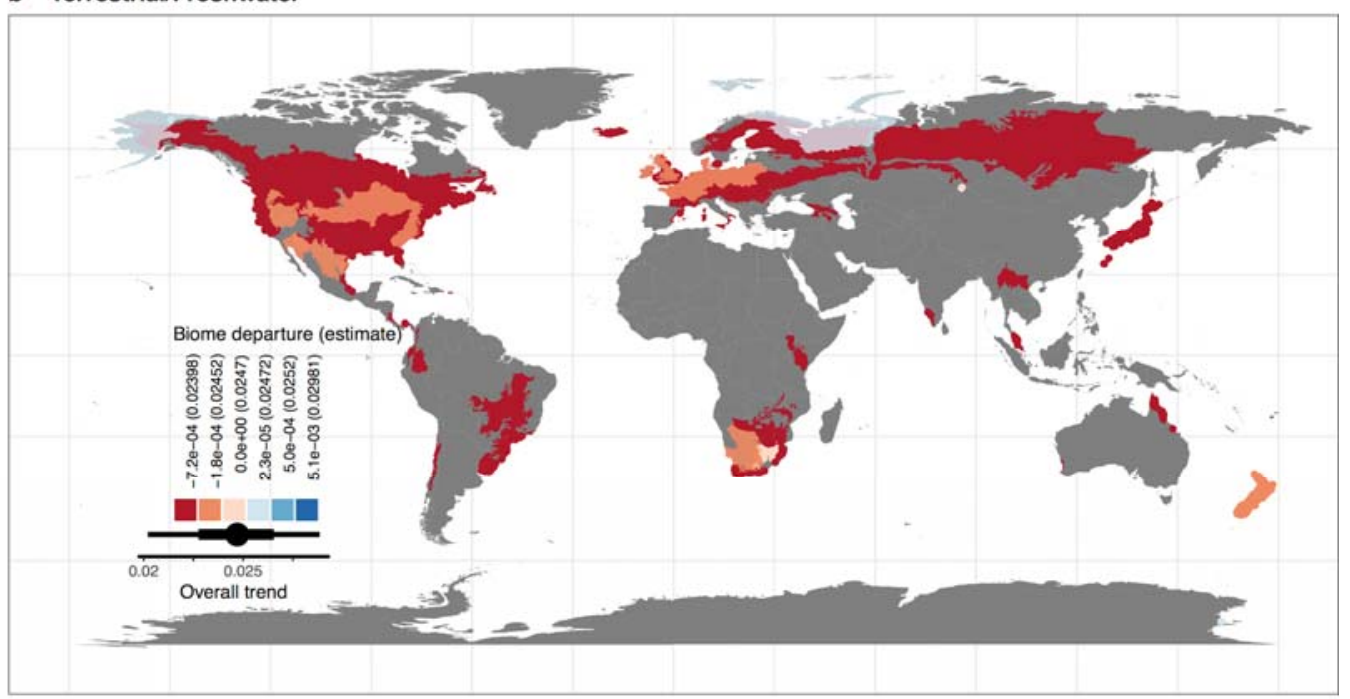

193 Figure 3. Assemblages across the globe are experiencing high rates of species replacement (turnover

194 component of Jaccard dissimilarity). a, Rates of new species replacing original species have both

195 positive and negative departures from the overall trend in marine biomes, whereas $\mathbf{b}$, terrestrial and

196 freshwater biomes have mostly slower rates of turnover than the overall trend (red shading). Numbers

197 in legend denote the departure and the biome-level (overall + departure) estimate in brackets. 
a
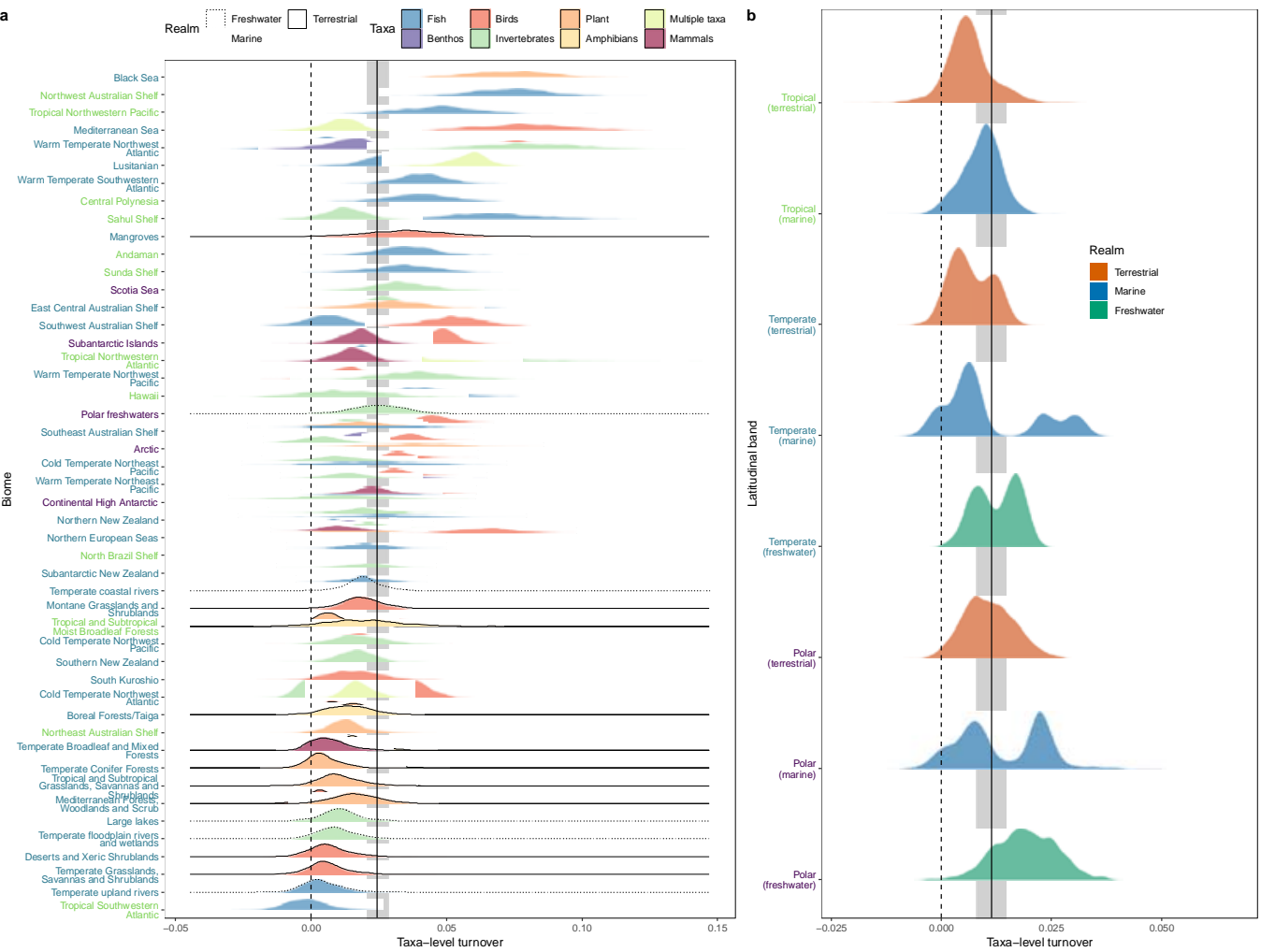

Figure 4. The magnitude and total variation of composition change (represented by the turnover

component of Jaccard dissimilarity) is greater among taxa in marine biomes compared to taxa in

204 terrestrial or freshwater biomes. The overall trend in turnover change per year is greater than zero

(solid black line; grey shading depicts the $90 \%$ credible interval) for both a, the biome-taxa model, and

206 b the simpler realm-climate-taxa model. Density ridges in a represent the posterior distribution for each

207 taxa-level slope coefficient (within each a biome); linetype and fill refer to the realm and taxon,

208 respectively. Colour of biome label denotes latitudinal band (light green tropical, blue green temperate

209 and purple polar as shown in b). Density ridges in $\mathbf{b}$ represent the posterior distributions of the slope

210 coefficients for all taxa within a given combination of realm and latitudinal band (climate) estimated

211 with the realm-climate-taxa model.

212 


\section{Linking richness and composition change}

215 To illustrate the relationship between trends in species richness and composition, we

216 plotted the dominant component of composition change (turnover or nestedness) for

217 each biome-taxa combination as a function of species richness change (Fig. 5a, b).

218 When turnover is the dominant component, this relationship shows how fast new

219 species are replacing original species, and whether or not these arrivals influence the

220 total number of species in assemblages. We found rates of turnover change to exceed

221 nestedness for more than $90 \%$ of biome-taxa combinations (97/105; Fig. 5b, c, d). For

222 these taxa, approximately one-third (31/97) had both rates of turnover and species

223 richness trends different from zero (Fig. 5c), with a balanced distribution of 14 cases

224 of species richness losses, and 17 with gains. When nestedness is the dominant

225 component, this relationship shows how fast assemblages are changing to become

226 smaller subsets of the species initially observed. Among the eight biome-taxa

227 combinations where nestedness exceeded turnover change (8/105; Fig. 5b, c, d), three

228 marine taxa had increasing nestedness and detectable species richness losses. Spatial

229 patterns of biodiversity change were highly heterogeneous (Fig. 5d), with locations in

230 close proximity experiencing distinct trends in composition and richness change. For

231 example, within the cold temperate northwest Atlantic marine biome (Fig. 5d,

232 Extended Data Figure 3; encompassing the Gulf of Maine, Gulf of St. Lawrence, and

233 south Newfoundland), the trend for invertebrate assemblages to become a smaller

234 subset of themselves was in close proximity to five other groupings of taxa where

235 turnover was the dominant component of composition change (benthos, birds, fish,

236 marine invertebrates/plants and multiple taxa), among which there were trends for

237 both species losses (e.g. birds) and gains (e.g. multiple taxa). 
a

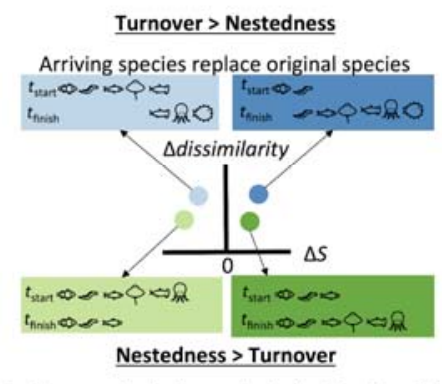

Arriving species independent of original species
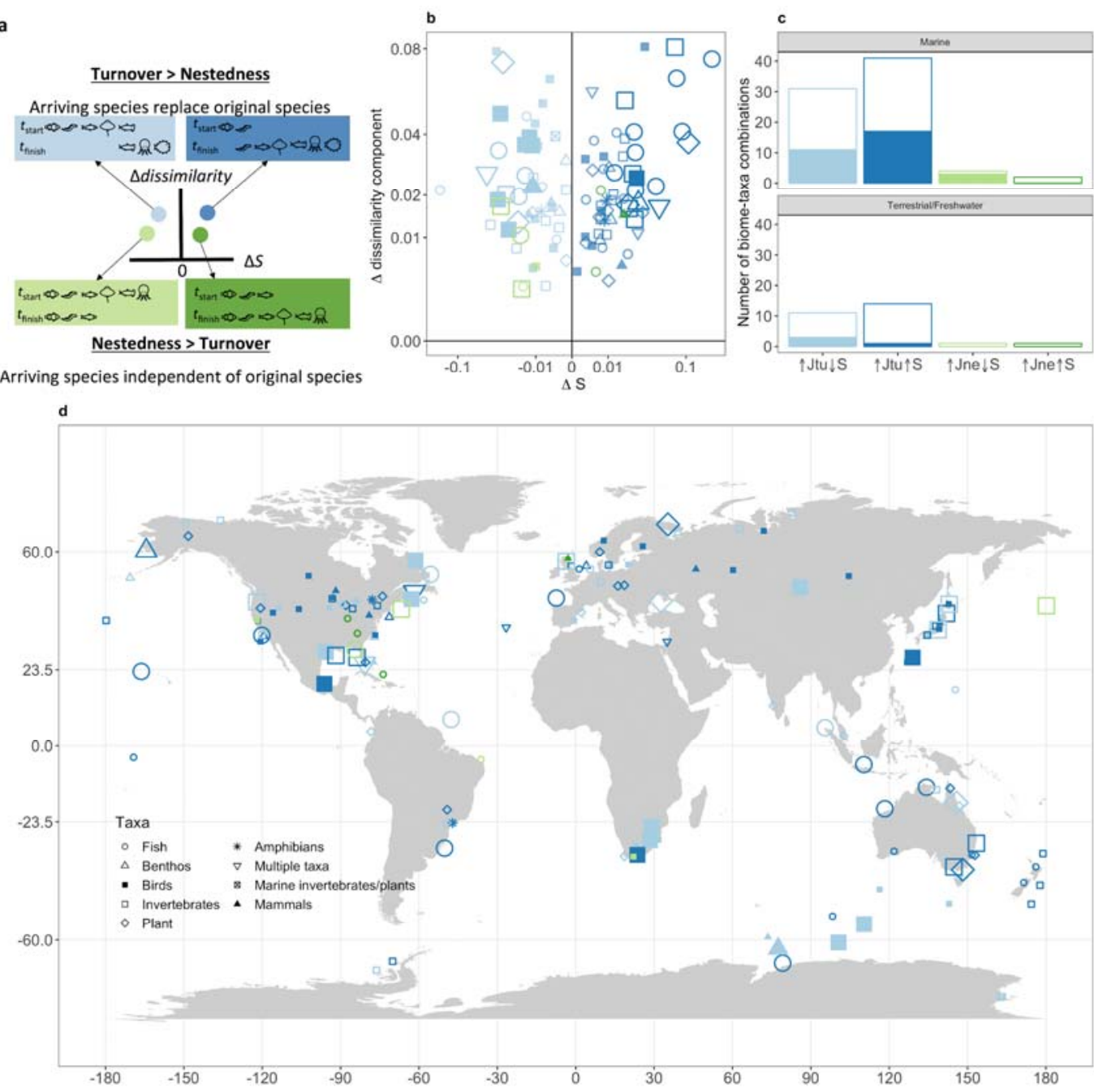

Figure 5. Conceptual and empirical relationships between changes in species richness and

composition. a, Conceptual model relating the turnover and nestedness components of species

243 composition change ( $\Delta$ dissimilarity) to changes in species richness $(\Delta S)$. When the turnover

244 component is larger than the nestedness component, new species entering assemblages replace the

245 original species (blue shaded boxes). Conversely, when the nestedness component is larger than the

246 turnover component, some original species of the assemblage remain, and the numbers of new species

247 entering the assemblage are largely independent of the original species (green shaded boxes). The

248 change in species richness documents the net change in the numbers of species in the assemblage (and

249 ignores their identity as either original or new species). b, Scatterplot showing the dissimilarity trend as

250 a function of the species richness trend. Each point represents a taxa-level group (shapes denoted in 
251 panel $\mathbf{d}$ legend), and larger points indicate that both composition and richness trends differed from zero

252 with 95\% probability; CIs not shown and axes transformed for clarity. c, The number of taxa for each

253 combination of change in species richness and species composition (measured as either the turnover,

254 Jtu, or nestedness, Jne, component of Jaccard dissimilarity). Filled sections of each bar represent taxa

255 where both species richness (S) and composition change (Jtu or Jne) differ from zero. d, Map showing

256 the location of each biome-taxa combination. Point colours and sizes follow from panel $\mathbf{b}$.

257

258 


\section{Discussion}

260 Compositional change dominated by species turnover is the most striking and most

261 prevalent pattern in biodiversity patterns across the globe. For the majority of taxa in

262 biomes across the planet for which we have data, there was considerable replacement

263 of species through time with no associated species richness changes. The consistent

264 pattern of species replacement is likely underpinned by a diverse suite of drivers.

265 Such reorganization is consistent with regulatory mechanisms for species richness.

266 Community regulation in species richness is widespread ${ }^{30}$, and may be driven by

267 shared resource availability ${ }^{29}$ or by the continuous replacement of transient

268 species $^{36,37}$. Contemporary pressures such as introduced species ${ }^{21-23}$, replacement of

269 localised specialists by widespread generalists, or range shifts in response to

270 environmental change ${ }^{18,38,39}$ may also help explain our finding of widespread

271 composition change and variable richness change with an overall trend not

272 distinguishable from zero.

273

274 Rates of species richness change and turnover were higher and more variable in the

275 marine realm. Detecting this difference between realms generates hypotheses about

276 the differences among realms in both drivers of biodiversity change and biotic

277 responses to these drivers. The spatial distributions of anthropogenic drivers of

278 change differ among realms ${ }^{17}$ and different driver combinations have different effects

279 on biodiversity change ${ }^{27}$. In addition, marine organisms may be more likely to

280 respond to some drivers such as climate change ${ }^{34}$ and marine assemblages may be

281 able to turnover more rapidly due to higher connectivity in the marine realm ${ }^{40}$. 
283 Amid widespread variation in biodiversity trends, we detect a signal of tropical

284 marine regions having a distribution of trends more skewed towards richness

285 extremes and high turnover. Particularly concerning are the two tropical marine

286 biomes that show both negative trends in species richness and higher than average

287 turnover of fish assemblages: Andaman and Tropical Northwestern Pacific. The

288 tropics, which harbour the majority of the planet's biological diversity, are generally

289 considered to be the most threatened regions of the planet ${ }^{41}$. Moreover, in the context

290 of climate change, there are likely fewer species available to replace species lost in

291 tropical zones that have entered no-analog warm temperature conditions ${ }^{42,43}$. If these

292 trends are maintained, this pattern could lead to dramatic global losses of biodiversity

293 and to the attenuation of the latitudinal diversity gradient, significantly altering the

294 planet's biogeography.

295

296 Here, we identify hotspots of biodiversity change: key areas that represent increasing

297 and decreasing extremes for biodiversity trends. In addition to the marine tropics,

298 areas like Tasmania, Alaska, and the South of South Africa stand out as regions

299 experiencing stronger negative biodiversity change. In contrast, the North Sea and

300 Eastern North America emerge as areas experiencing increases in biodiversity change.

301 This spatial and taxonomic variation in biodiversity trends means that global trends of

302 biodiversity need to be based on spatially representative data. However, and despite

303 using the largest compilation of biodiversity time series to date, our analysis suffers

304 from many blind-spots. Our results highlight how improving our understanding of

305 biodiversity change will require filling the gaps by improving biodiversity monitoring

306 and moving towards global stratified random sampling of biodiversity. 
308 In summary, biodiversity change has strong biogeographic variation. We have

309 identified hotspots of richness gains and losses, as well as species replacement. The

310 marine realm emerges as having the strongest change, and the marine tropics in

311 particular, as having a higher prevalence of richness losses. This spatial variation

312 suggests we need to abandon a view of homogenous loss of biodiversity, as the mean

313 of local change across the globe does not differ from zero, and is not necessarily

314 representative of local trends. Our work suggests an urgent need to better understand

315 why there is such geographic variation. The spatial variation described here should

316 inform conservation prioritisation by identifying the parts of the planet changing most

317 rapidly, as well as those that are more stable. In the field of climate science, there was

318 a shift in wording from global warming to climate change. Similarly, our results

319 justify a shift in focus towards recognising that biodiversity change in the

320 Anthropocene has contrasting effects in different parts of the planet.

321 Methods

\section{Data description and pre-processing}

323 The BioTIME database represents the largest global effort mobilizing assemblage

324 time series (range $=2$ to 97 years; mean $=5.5$ years), includes 386 studies, and

325 currently holds over 12 million records of abundance for over 45 thousand species

326 across plants, invertebrates, fish, birds and mammals ${ }^{28}$. Analyses presented in this

327 study are based on time series of abundance data (i.e., the studies that recorded counts

328 of the number of individuals for each species in an assemblage).

330 As we were interested in quantifying biodiversity change at the local scale, studies

331 with multiple sampling locations and extents greater than $71.7 \mathrm{~km}^{2}$ were partitioned 
332 into $96 \mathrm{~km}^{2}$ grid cells (studies with extents $<71.7 \mathrm{~km}^{2}$ were assigned to the grid cell

333 in which they were centered). Each cell was given a unique identifier that was the

334 concatenation of the study ID and the cell reference number. Species were then

335 collated within each grid cell for each year, resulting in new assemblage time series

336 within grid cells. For all assemblages in every cell and every year, we calculated the

337 coverage of the sample, which is a measure of sample completeness ${ }^{44}$ (mean $=0.95$,

$338 \mathrm{sd}=0.11$ ), and removed all assemblage-cell-year combinations with coverage less

3390.85 (meaning that for the time series retained, there was a $<15 \%$ chance of another

340 individual sampled being a new species).

342 Finally, we applied sample-based rarefaction to the minimum number of samples per

343 year for each time series. We calculated community dissimilarity using pairwise

344 Jaccard dissimilarity and the first year as the baseline, and species richness for each

345 year of the time series. We also calculated the turnover and nestedness components of

346 Jaccard dissimilarity to assess if the changes in compositional diversity were driven

347 by species replacement or changes in species richness ${ }^{35,45}$.

\section{Models of biodiversity change}

350 We quantified biodiversity change using two complementary hierarchical models.

351 Both models included the time series of assemblage dynamics within individual cells

352 at the lowest level of the model, but differed in the way that these individual time

353 series were grouped hierarchically. The biome-taxa (BT) model nested time series

354 within ecological biomes (http://maps.tnc.org/gis_data.html ${ }^{46-49}$ ) and groups of taxa.

355 The realm-climate-taxa (RCT) model nested time series within a grouping covariate

356 that was the concatenation of realm (marine, terrestrial or freshwater), climate 
357 (latitudinal bands denoting polar regions, temperate regions and the tropics) and taxa.

358 The groupings of taxa for both models were based on the metadata of BioTIME, and

359 included: amphibians, benthos, birds, fish, invertebrates, mammals, marine

360 plants/invertebrates, plants, and multiple taxa for studies that measured more than one

361 taxa group. We fit models where there were more than three cell-level times series per

362 group, and discuss trends at the biome and taxa levels for the BT model, and for the

363 realm-climate-taxa level for the RCT model, as the analytic technique is not well

364 suited to describing trends at the cell-level where the data are sparse. Both models

365 were fit with year as a population (or global) parameter, and year (i.e., the slope

366 parameter) and the intercept were allowed to vary for each of the hierarchical levels of

367 the models.

369 We quantified change in species richness and community composition (total Jaccard

370 dissimilarity, and the turnover and nestedness components). Species richness was

371 modelled assuming a Poisson error distribution and a log link function. This resulted

372 in the BT model having the form:

$$
\log \left(\mu_{k, j, i, t}\right)=\beta_{0}+\beta_{0 k}+\beta_{0 k, j}+\beta_{0 k, j, i}+\left(\beta_{1}+\beta_{1 k}+\beta_{1 k, j}+\beta_{1 k, j, i}\right) x_{k, j, i, t},
$$

$$
y_{k, j, i, t} \sim \operatorname{poisson}\left(\mu_{k, j, i, t}\right)
$$

376 where $x_{k, j, i, t}$ is the time in years, $\beta_{0}$ and $\beta_{1}$ are the global intercept and slope (often

377 termed fixed effects), $\beta_{0 k}$ and $\beta_{1 k}$ are the biome-level departures from $\beta_{0}$ and $\beta_{1}$

378 (respectively; biome-level random effects), $\beta_{0 k, j}$ and $\beta_{1 k, j}$ are taxa-level departures

379 (nested within biomes) from $\beta_{0}$ and $\beta_{1}$ (taxa-level random effects), $\beta_{0 k, j, i}$ and $\beta_{1 k, j, i}$ are 
380 the (nested) cell-level departures from $\beta_{0}$ and $\beta_{l}$ (cell-level random effects); $y_{k, j, i, t}$ is

381 the (rarefied) species richness in year $t$ of the $i$ th cell for the $j$ th taxonomic group

382 within the $k$ th biome.

383

384 The species richness RCT model had the form:

385

$$
\log \left(\mu_{j, i, t}\right)=\beta_{0}+\beta_{0 j}+\beta_{0 j, i}+\left(\beta_{1}+\beta_{1 j}+\beta_{1 j, i}\right) x_{j, i, t}
$$

386

$$
y_{j, i, t} \sim \operatorname{poisson}\left(\mu_{j, i, t}\right)
$$

388 where $x_{j, i, t}$ is the time in years, $\beta_{0}$ and $\beta_{1}$ are the global intercept and slope (fixed

389 effects), $\beta_{0 j}$ and $\beta_{1 j}$ are the departures for each realm-climate-taxa group from $\beta_{0}$ and

$390 \beta_{1}$ (respectively; random effects), $\beta_{0 j, i}$ and $\beta_{1 j, i}$ are the cell-level departures from $\beta_{0}$ and

$391 \beta_{1}$ (cell-level random effects); $y_{j, i, t}$ is the (rarefied) species richness in year $t$ of the $i$ th

392 cell, of the $j$ th combination of realm-climate-taxa.

394 All dissimilarity metrics were modelled assuming Gaussian error and an identity link 395 function, resulting in BT models of the form:

396

$$
\mu_{k, j, i, t}=\beta_{0}+\beta_{0 k}+\beta_{0 k, j}+\beta_{0 k, j, i}+\left(\beta_{1}+\beta_{1 k}+\beta_{1 k, j}+\beta_{1 k, j, i}\right) x_{k, j, i, t}
$$

$$
y_{k, j, i, t} \sim N\left(\mu_{k, j, i, t}, \sigma^{2}\right)
$$

399 where $x_{k, j, i, t}$ is the time in years, $\beta_{0}$ and $\beta_{1}$ are the global intercept and slope, $\beta_{0 k}$ and $\beta_{1 k}$

400 are the biome-level departures from $\beta_{0}$ and $\beta_{1}$ (respectively), $\beta_{0 k, j}$ and $\beta_{1 k, j}$ are the taxa- 
401 level departures from $\beta_{0}$ and $\beta_{1}, \beta_{0 k, j, I}$ and $\beta_{1 k, j, I}$ are the cell-level departures from $\beta_{0}$

402 and $\beta_{1}$, and $y_{k, j, i, t}$ is the value of the dissimilarity metric (total Jaccard dissimilarity, or

403 one of the components) in year $t$ of the $i$ th cell, of the $j$ th taxonomic group within the

$404 k$ th biome. The dissimilarity metric was set to equal zero (perfectly similarity) for the

405 first year of each time series.

406

407 The dissimilarity RCT models had the form:

408

$$
\mu_{j, i, t}=\beta_{0}+\beta_{0 j}+\beta_{0 j, i}+\left(\beta_{1}+\beta_{1 j}+\beta_{1 j, i}\right) x_{j, i, t}
$$

409

$$
y_{j, i, t} \sim N\left(\mu_{j, i, t}, \sigma^{2}\right)
$$

410

411 where $x_{j, i, t}$ is the time in years, $\beta_{0}$ and $\beta_{1}$ are the global intercept and slope, $\beta_{0 j}$ and $\beta_{l j}$

412 are the departures for each realm-climate-taxa group from $\beta_{0}$ and $\beta_{1}$ (respectively),

$413 \beta_{0 j, i}$ and $\beta_{1 j, i}$ are the cell-level departures from $\beta_{0}$ and $\beta_{l}$, and $y_{j, i, t}$ is the value of the

414 dissimilarity metric in year $t$ of the $i$ th cell, of the $j$ th combination of realm-climate-

415 taxa. The dissimilarity metric was set to equal zero (perfectly similarity) for the first

416 year of each time series.

417

418 We used weakly regularising normally-distributed priors for the global intercept and

419 slope:

420

$$
\begin{aligned}
& \beta_{0} \sim N(0,5), \\
& \beta_{1} \sim N(0,2),
\end{aligned}
$$


422 Group-level parameters were all assumed to be $N(0, \sigma)$, and priors on the $\sigma$ were the

423 same for all models of composition (the turnover and nestedness components of

424 Jaccard's dissimilarity) and the RCT of species richness (i.e., as follows, with the $k$

425 subscript dropped for the RCT models):

426

$$
\begin{aligned}
& \sigma_{\beta_{0 k}}=\sigma_{\beta_{0 k, j}}=\sigma_{\beta_{0 k, j, i}} \sim \text { half Cauchy }(0,2.5), \\
& \sigma_{\beta_{1 k}}=\sigma_{\beta_{1 k, j}}=\sigma_{\beta_{1 k, j, i}} \sim \operatorname{half} \text { Cauchy }(0,2.5) .
\end{aligned}
$$

428 The group-level parameters of the BT species richness model were also assumed to be $N(0, \sigma)$, but the priors were drawn from the student $t$ distribution:

$$
\begin{aligned}
& \sigma_{\beta_{0 k}}=\sigma_{\beta_{0 k, j}}=\sigma_{\beta_{0 k, j, i}} \sim \text { student } t(1,0,10), \\
& \sigma_{\beta_{1 k}}=\sigma_{\beta_{1 k, j}}=\sigma_{\beta_{1 k, j, i}} \sim \operatorname{student} t(1,0,10) .
\end{aligned}
$$

432 Correlations between levels of the grouping-factors (e.g., taxa with biomes) are 433 estimated using the Cholesky decomposition (L) of the correlation matrix, with a 434 Lewandowski-Dorota-Joe (LKJ) prior ${ }^{50}$, here set as:

$$
L \sim L K J(2)
$$

437 Model convergence and goodness of fit were assessed using a combination of 438 statistics (Gelman-Rubin diagnostic ${ }^{51,52}$ ) and visual inspection of the Markov chains. 
440 All data manipulation and analysis were conducted in $\mathrm{R}\left(3.3 .1\right.$ or greater $\left.{ }^{53}\right)$. Models

441 were coded using the 'brms' package (version 1.5.1 or greater ${ }^{50}$ ), which fits models

442 with the probabilistic programming language $\operatorname{Stan}^{50}$.

\section{Sensitivity analyses}

444 A recurrent criticism of existing time series analyses is the lack of an appropriate

445 baseline from which to detect change ${ }^{11,54}$. Obtaining baselines for all of the datasets in

446 the BioTIME database is unrealistic, but we assessed whether the rates of change are

447 themselves changing through time by quantifying biodiversity change for different

448 time periods (since the 1950's). To do this, we subset the data into three periods:

449 1951-1970, 1971-1990, and 1990-2010, and refit each of the models to each of these

450 subsets (Extended Data Figs. 4-6).

451

452 We also assessed the sensitivity of our estimates of biodiversity change to the length

453 of the time series (number of discrete years sampled), and the starting year of each

454 time series (Extended Data Fig. 10). Additionally, we examined the estimates of

455 change as a function of the initial assemblage species richness (i.e., the number of

456 species in the first year of each assemblage time series; Extended Data Fig. 10).

457

458 Code availability

459 Code is available on an online archive at Zenodo: (doi:

460 https://doi.org/10.5281/zenodo.1475218)

461

462

463 Data availability

464 The time series analysed were from 332 unique references found in the BioTIME

465 dataset and in other studies which were used with permission. 
467 Approximately $92 \%$ (306 references) of the biodiversity studies analysed here are

468 available as part of the published BioTIME Database ${ }^{28}$. The data are openly available,

469 and can be accessed on Zenodo (https://doi.org/10.5281/zenodo.1211105) or through

470 the BioTIME website (http://biotime.st-andrews.ac.uk/).

471

472 Dornelas, M., Antão, L.H., Moyes, F., Bates, A.E., Magurran, A.E., and BioTIME

473 consortium (200+ authors). 2018. BioTIME: a database of biodiversity time-series

474 for the Anthropocene. Global Ecology and Biogeography. 10.1111/geb.12729

475

476 The remaining $8 \%$ (26 references) of biodiversity studies analysed were used with

477 permission. Some of these studies are published and publicly available outside of the

478 BioTIME database, and others are available with permission from the corresponding

479 author on reasonable request. For more details regarding the original citations,

480 corresponding authors, and availability of these datasets, please refer to Table S1 in

481 Dornelas et al. (2018).

\section{References}

4831 Pimm, S. L. et al. The biodiversity of species and their rates of extinction,

484 distribution, and protection. Science 344, doi:10.1126/science.1246752 (2014).

4852 Proença, V. \& Pereira, H. M. in Encyclopedia of Biodiversity (Second Edition)

486 (ed Simon A. Levin) 167-176 (Academic Press, 2013).

4873 Newbold, T. et al. Global effects of land use on local terrestrial biodiversity.

$488 \quad$ Nature 520, 45, doi:10.1038/nature14324

489 https://www.nature.com/articles/nature14324\#supplementary-information (2015).

4904 García Molinos, J. et al. Climate velocity and the future global redistribution

491 of marine biodiversity. Nature Clim. Change 6, 83-88,

492 doi:10.1038/nclimate2769

493 http://www.nature.com/nclimate/journal/v6/n1/abs/nclimate2769.html\#supplementary

$494 \quad$-information (2016). 
54423 Winter, M. et al. The role of non-native plants and vertebrates in defining

545 patterns of compositional dissimilarity within and across continents. Global

$546 \quad$ Ecology and Biogeography 19, 332-342, doi:10.1111/j.1466-

$547 \quad$ 8238.2010.00520.x (2010).

54824 Parmesan, C. \& Yohe, G. A globally coherent fingerprint of climate change

549 impacts across natural systems. Nature 421, 37, doi:10.1038/nature01286

550 https://www.nature.com/articles/nature01286\#supplementary-information (2003).

$55125 \quad$ Lenoir, J. \& Svenning, J. C. Climate-related range shifts - a global

552

553

554

555

556

557

558

559

multidimensional synthesis and new research directions. Ecography 38, 15-28, doi:10.1111/ecog.00967 (2015).

26 Fahrig, L. Ecological responses to habitat fragmentation per se. Annual

Review of Ecology, Evolution, and Systematics 48, 1-23 (2017).

27 Dunic, J. C. et al. Attributing the variability in direction and magnitude of local-scale marine biodiversity change to human activities. bioRxiv, doi:10.1101/162362 (2017).

28 Dornelas, M. et al. BioTIME: A database of biodiversity time series for the

560

561

562

563

564

565

566

567

568

569

570

571

572

573

574

575

576

577

578

579

580

581

582 Anthropocene. Global Ecology and Biogeography 27, 760-786,

doi:10.1111/geb.12729 (2018).

29 Brown, J. H., Ernest, S. M., Parody, J. M. \& Haskell, J. P. Regulation of diversity: maintenance of species richness in changing environments. Oecologia 126, 321-332 (2001).

30 Gotelli, N. J. et al. Community-level regulation of temporal trends in

biodiversity. Science Advances 3, doi:10.1126/sciadv.1700315 (2017).

31 MacArthur, R. H. \& Wilson, E. O. The Theory Of Island Biogeography. (Princeton University Press, 1967).

32 Magrin, G. et al. Central and South America in Climate Change 2014: Impacts, Adaptation, and Vulnerability. Part B: Regional Aspects. Contribution of Working Group II to the Fifth Assessment Report of the Intergovernmental Panel of Climate Change (eds. Barros, VR et al.), 14991566 (2014).

33 Wiens, J. J. Climate-Related Local Extinctions Are Already Widespread among Plant and Animal Species. PLOS Biology 14, e2001104, doi:10.1371/journal.pbio.2001104 (2016).

34 Sunday, J. M., Bates, A. E. \& Dulvy, N. K. Thermal tolerance and the global redistribution of animals. Nature Climate Change 2, 686, doi:10.1038/nclimate1539

https://www.nature.com/articles/nclimate1539\#supplementary-information (2012).

35 Baselga, A. Partitioning the turnover and nestedness components of beta diversity. Global Ecology and Biogeography 19, 134-143, doi:10.1111/j.1466-

583

584 8238.2009.00490.x (2010).

585

36 Magurran, A. E. \& Henderson, P. A. Explaining the excess of rare species in

586

587 natural species abundance distributions. Nature 422, 714, doi:10.1038/nature01547 (2003).

37 Snell Taylor, S. J., Evans, B. S., White, E. P. \& Hurlbert, A. H. The prevalence and impact of transient species in ecological communities. Ecology 99, 1825-1835, doi:10.1002/ecy.2398 (2018). 


\begin{tabular}{|c|c|c|}
\hline 592 & 39 & Batt, R. D., Morley, J. W., Selden, R. L., Tingley, M. W. \& Pinsky, M. L. \\
\hline 593 & & Gradual changes in range size accompany long-term trends in species \\
\hline 4 & & richness. Ecology Letters, n/a-n/a, doi:10.1111/ele.12812 (2017). \\
\hline 95 & 40 & Kinlan, B. P. \& Gaines, S. D. Propagule dispersal in marine and terrestrial \\
\hline 96 & & vironments: a community perspectiv \\
\hline 97 & 41 & Barlow, J. et al. The future of hyperdiverse tropical ecosystems. Nature $\mathbf{5 5 9}$, \\
\hline 8 & & 517-526, doi:10.1038/s41586-018-0301-1 (2018). \\
\hline 99 & 42 & Burrows, M. T. et al. Geographical limits to species-range shifts are suggested \\
\hline 0 & & by climate velocity. Nature $\mathbf{5 0 7}, 492-49$ \\
\hline 01 & 43 & Pinsky, M. L., Worm, B., Fogarty, M. J., Sarmiento, J. L. \& Levin, S. A. \\
\hline 02 & & Marine taxa track local climate velocities. Science 341, 1239-1242, \\
\hline & & doi:10.1126/science.1239352 (2013). \\
\hline 14 & 44 & Chao, A. \& Jost, L. Coverage-based rarefaction and extrapolat \\
\hline 05 & & standardizing samples by completeness rather than size. Ecology $\mathbf{9 3}$, \\
\hline 66 & & 2547, doi:10.1890/11-1952.1 (2012). \\
\hline & 45 & $\begin{array}{l}\text { Baselga, A. et al. betapart: Partitioning beta diversity into turnover and } \\
\text { nestedness }\end{array}$ \\
\hline 99 & & nents. $R$ package version 1.5.1, https://cran.r- \\
\hline 10 & & project.org/web/packages/betapart/betapart.pdf (2018). \\
\hline 11 & 46 & TNC. terrestrial ecoregions dataset: The Nature Conservancy. \\
\hline & & tnc_terr_ecoregions. The Nature Conservancy, Arlington, VA, vector digital \\
\hline 13 & & data. 14 December 2009. http://maps.tnc.org (2009). \\
\hline 14 & 47 & Abell, R. et al. Freshwater ecoregions of the world: a new map of \\
\hline 15 & & er biodiversity conservation. AIBS \\
\hline 16 & & $\mathbf{5 8}, 403-414(2008)$ \\
\hline 17 & 48 & Olson, D. M. \& Dinerstein, E. The Global 200: Priority ecoregions for global \\
\hline 18 & & uri Botanical garden, 199-224 (2002). \\
\hline 19 & 49 & Spalding, M. D. et al. Marine Ecoregions of the World: A Bioregionalization \\
\hline & & of Coastal and Shelf Areas. BioScience 57, 573-583, doi:10.1641/B570707 \\
\hline 21 & & (2007). \\
\hline 22 & 50 & Bürkner, P.-C. brms: An R Package for Bayesian Multilevel Models Using \\
\hline 2. & & 201780,2 \\
\hline 2 & 51 & Gelman, A. \& Rubin, D. B. Inference from iterative simulation using multiple \\
\hline 62 & & sequences. Statistical science 7, 457-472 (1992). \\
\hline 26 & 52 & Gelman, A. et al. Bayesian data analysis. (Chapman and Hall/CRC, 2013). \\
\hline 62 & 53 & R: A Language and Environment for Statistical Computing (R Foundation for \\
\hline 6 & & Statistical Computing, http://www.R-project.org/, Vienna, Austria, 2018). \\
\hline 629 & 54 & Cardinale, B. J., Gonzalez, A., Allington, G. R. H. \& Loreau, M. Is local \\
\hline & & biodiversity declining or not? A summary of the debate over analysis of \\
\hline & & cies richness time trends. Biological Con \\
\hline & & doi:https://doi.org/10.1016/j.biocon.2017.12.021 (2018). \\
\hline
\end{tabular}

\section{Acknowledgements}

636 We thank all the authors who contributed their data, or gave us permission to include

637 it in this study. Mary O'Connor helped in securing the funding for the working group 
638 and contributed to discussions and editing the manuscript drafts. This work was

639 supported by funding to the sChange working group through sDiv, the synthesis

640 centre of iDiv, the German Centre for Integrative Biodiversity Research Halle-Jena-

641 Leipzig, funded by the German Research Foundation (FZT 118). Many working

642 group members contributed to the projects in various roles across multiple meetings,

643 and were influential in shaping the discussions and ideas that led to this paper. Those

644 not listed as authors here include: Mary O'Connor, Juliano Sarmento Cabral, Jillian

645 Dunic, and Robin Elahi. SB, HB, JMC, JH and MW were supported by the German

646 Centre for Integrative Biodiversity Research (iDiv) Halle-Jena-Leipzig. SRS was

647 supported by National Science Foundation grant 1400911. LHA was supported by

648 Fundação para a Ciência e Tecnologia, Portugal (POPH/FSE SFRH/BD/90469/2012).

649 MD was supported by a Leverhulme Trust Fellowship. AEM, FM and MD were

650 supported by ERC AdG BioTIME 250189 and PoC BioCHANGE 727440.

\section{Author Information}

652 These authors contributed equally and led the paper: Shane Blowes and Sarah Supp.

653 Maria Dornelas was the senior author on the paper.

\section{Author Contributions}

655 MD conceived the project. SB, SRS, and MD led the development of the project,

656 assisted with data analysis and interpretation, and wrote the first draft of the

657 manuscript. SB and SRS collaborated on the core data preparation and coding the

658 analysis in R. SB designed the analytical models and prepared the figures for the

659 manuscript. FM managed the BioTIME database, queried it for the analysis, and

660 provided help with figures. All authors contributed to the sDiv working group that

661 conceived this project, to key discussions that led to the design of the study, and 
662 helped with revising the paper and preparing and approving it for publication. MD

663 and SRS secured the funding from sDiv and co-led the sChange working group (with

664 Mary O'Connor) that initiated the project.

665

666 Competing Interests

667 The authors declare no competing interests.

668

669 Corresponding Author

670 Correspondence and requests for materials should be addressed to the lead authors:

671 Shane Blowes (ablowes@gmail.com), Sarah Supp (supps@ denison.edu), and Maria

672 Dornelas (maadd@st-andrews.ac.uk).

673 Supplementary Information

674 Further information detailing the results, including the sensitivity analysis, is available

675 in the Supplementary Information and Extended Data files, linked to this paper.

676 